\title{
Lesion aspirate culture for the diagnosis and isolation of Leishmania spp. from patients with cutaneous leishmaniasis
}

\author{
Zélia Maria Profeta Luz/ ${ }^{+}$, Alexandre Rotondo da Silva, Fernanda de Oliveira Silva, \\ Rachel B Caligiorne, Edward Oliveira, Ana Rabello
}

Laboratório de Pesquisas Clínicas, Centro de Referência em Leishmanioses, Instituto René Rachou-Fiocruz, Av. Augusto de Lima 1715, 30190-002 Belo Horizonte, MG, Brasil

The detection of Leishmania spp. in skin lesion aspirates, using a puncture technique, was evaluated in 76 patients with cutaneous leishmaniasis (CL) who were referred to a Leishmaniasis Reference Centre in Brazil. CL was defined based on skin lesions suggestive of the disease and on a positive result of the Montenegro skin test or Giemsa-stained imprints of biopsy fragments. The aspirates were cultured using a vacuum tube device containing culture medium and evaluated for the presence of Leishmania spp. The biphasic medium culture was examined once a week for three weeks. Promastigotes were observed in 53/76 (69.7\%) cultures. Stained smears from 60 of the 76 patients were evaluated using PCR-RFLP to detect the conserved minicircle region of Leishmania spp. and to classify the parasite. Of these patients, $45(75 \%)$ showed positive results in aspirate culture and 15 presented negative results. The PCR was positive in 80\% (53/60) samples. The PCR-RFLP profile was determined in 49 samples, of which 45 (92\%) showed a pattern compatible with Leishmania (Viannia) braziliensis. The aspirate culture is a sensitive and feasible method for diagnosing $C L$ and may be routinely adopted by health services for $\mathrm{L}$. (V.) braziliensis isolation and identification.

Key words: puncture biopsy - cutaneous leishmaniasis - diagnosis - health services

American tegumentary leishmaniasis (ATL) is a zoonotic disease caused by parasites belonging to the Leishmania genus. The disease encompasses a broad range of severities and clinical manifestations and can be present as cutaneous or mucosal leishmaniasis (MS 2007). The clinical characteristics of ATL differ depending on the infecting Leishmania species and the host immune response.

In Brazil, as in other parts of Latin America, this disease was originally associated with forested areas, but has now adapted to the urban environment due to deforestation and disordered urbanization (Reithinger \& Davies 1999, Desjeux 2004).

Currently, approximately 30,000 new cases of ATL are reported to the Brazilian Ministry of Health annually. Most of these cases are classified as cutaneous leishmaniasis (CL). The main species implicated in the transmission are Leishmania (Viannia) braziliensis and Leishmania (Leishmania) amazonensis, which are distributed throughout the country, and Leishmania (Viannia) guyanensis, which is concentrated north of the Amazon River, in the Northern region of Brazil (Grimaldi et al. 1989, Romero et al. 2001). The control of leishmaniasis has been difficult due to the diversity of Leishmania species reservoirs and the vectors involved in disease transmission.

Financial support: FIOCRUZ, CAPES, CNPq

+ Corresponding author: profeta@cpqrr.fiocruz.br

Received 28 July 2008

Accepted 5 January 2009
The diagnosis of CL based on clinical aspects has low accuracy since other conditions such as fungal and mycobacterial infections, sarcoidosis and neoplasms can mimic CL infection. Serological and histopathological methodologies have limited diagnostic accuracy, due to their low sensitivity. The cell-mediated immune response measured by delayed-type hypersensitivity usually identifies positive cases accurately. However, results can be dependant upon the immunological status of the patient, the time since infection, the infective species of Leishmania and the quality of the reagents used. In addition, despite the high specificity of the skin test, it cannot distinguish between current and past infections (Marzochi et al. 1993, Berman 1997).

Molecular tools such as PCR have been shown to be highly effective in the diagnosis of leishmaniasis (Reed 1996, Pirmez et al. 1999, Weigle et al. 2002, Disch et al. 2005). However, the drawbacks to this approach include the cost, the availability of reagents and equipment and the poor adaptability to field conditions.

The currently used parasitological diagnostic methodology involves the direct observation of amastigotes in stained smears or cultures of material obtained from skin lesions. The traditional culture methods consist of a biphasic culture system of blood agar with liquid overlay (Marzochi et al. 1993, Berman 1997). Simpler procedures for obtaining lesion material from CL patients are needed, since the complexity of the collection significantly restricts its large-scale use in public health settings. CL lesion aspirate cultivation constitutes one alternative methodology since it is easily performed and has a high positivity rate, ranging from $28.6-89 \%$ in studies that evaluated patients infected with different 
Leishmania species (Salinas et al. 1989, Marzochi et al. 1993, Romero et al. 1999, Weigle et al. 2002).

In this work, we assessed the detection rate of Leishmania spp. in skin lesion aspirate cultures performed in patients with CL in the Southeast region in Brazil

\section{PATIENTS, MATERIAL AND METHODS}

Patients - Samples were taken from the lesions of 76 patients residing in the metropolitan region of Belo Horizonte, Minas Gerais (MG), Brazil, who were referred to the Leishmaniasis Reference Centre of the René Rachou Institute-Fiocruz, from October 2003-October 2005. All patients with skin lesions suggestive of $C L$ were invited to participate. After written informed consent was given, all patients underwent the Montenegro skin test, biopsy collection and lesion aspiration. The criteria for inclusion in the study was the presence of skin lesions suggestive of CL and one positive result from either the Montenegro skin test or Giemsa-stained imprints of biopsy fragments. The criteria for exclusion were the presence of clinically manifested secondary infections, more than six cutaneous ulcers and patients with previous specific treatment. All patients received specific treatment for $\mathrm{CL}$ as recommended by the Brazilian Ministry of Health guidelines (MS 2007).

Skin test - For the Montenegro skin test, an antigen suspension composed of killed L. (L.) amazonensis promastigotes was used, according to the manufacturer's instructions (Biomanguinhos-Fiocruz). The antigen was applied to the volar surface of the forearm and the reaction was measured after 48-72 h (Sokal 1975). An induration of $\geq 5 \mathrm{~mm}$ was considered positive.

Stained smear - A fragment from the ulcer border was obtained by lesion biopsy using a $3.5 \mathrm{~mm}$ punch. For each fragment, two slides were prepared with eight imprints each. The slides were fixed with methanol, stained with Giemsa and observed by oil immersion microscopy (100X).

Aspirate culture preparation - The aspirate culture was performed according to Marzochi et al. (1993) and Romero et al. (1999), with minor modifications. For the culture, $10 \mathrm{~mL}$ capacity vacuum tubes (Vacutainer, Becton, Dickinson and Company, São Paulo, SP, Brazil) with rubber caps were filled with a biphasic culture medium. The solid phase was composed of $2 \mu \mathrm{L}$ of blood agar base - NNN medium Sigma (Sigma-Aldrich, St Louis, MO, USA), with $10 \%$ defribinated rabbit blood added after hardening of the agar at $50^{\circ} \mathrm{C}$ (Chang \& Fish 1983). The liquid phase was composed of $200 \mu \mathrm{L}$ isotonic saline $(0.9 \%)$, containing $100 \mathrm{Units} / \mathrm{mL}$ penicillin and 100 $\mu \mathrm{g} / \mathrm{mL}$ streptomycin (Pen/Strep Gibco, Grand Island, NY, USA). Tubes were recapped and the vacuum was restored by aspirating $15 \mathrm{~mL}$ of air from the tube twice with a $20 \mathrm{~mL}$ syringe equipped with a 22 gauge needle.

Puncture procedure and cultivation - The puncture site was vigorously cleaned with $70 \%$ ethanol and a local anaesthetic [(100 $\mu \mathrm{L}$ of $2 \%$ lidocaine) Hipofarma, Brazil] was injected with a $1 \mathrm{~mL}$ syringe equipped with a $13 \mathrm{G}$ needle. The aspiration puncture was made using a commercially available Vacutainer needle holder and a $21 \mathrm{G}$ needle was inserted into the dermis of the ulcer border, at a $45^{\circ}$ angle, with a rotary movement. Under suction, the material was transferred to the vacuum tube containing the culture medium. The needle was removed with the tube connected, allowing air to enter due to air pressure. For each patient, the procedure was performed at the same lesion site three times with different tubes.

The culture tubes were incubated (FANEM BOD $347 \mathrm{CD}$ ) at $26^{\circ} \mathrm{C}$ for 21 days. For parasite examination, the contents of each tube were gently homogenized and approximately $10 \mu \mathrm{L}$ of the suspension was observed using an optical microscope (40X magnification, approximately 150 fields) at seven, 14 and 21 days postaspiration. When identified as positive, the culture was transferred to a culture flask containing NNN plus LIT medium (Liver Infusion Tryptose, DIFCO) for expansion and to obtain parasites for cryopreservation.

Isolation of DNA from imprint slides - Sixty milliliter of sterile water were used to re-suspend three Giemsastained imprints from the stored slide of each patient. The volume was transferred to a microtube and heated at $95^{\circ} \mathrm{C}$ for $15 \mathrm{~min}$ and then centrifuged at $13,000 \mathrm{~g}$. The supernatant was collected and stored at $-20^{\circ} \mathrm{C}$ until use.

PCR amplification - PCR was performed using the following oligonucleotides: (i) a sense genus-specific primer (GFP: GGG TAG GGG CGT TCT GCG AA) and (ii) a 1:1 mixture of anti-sense sub-genus specific primers (GRP $(V$.$) : GGC CCA CTA TAT TAC ACC AAC$ CCC and GRP(L.): CCG CCC CTA TTT TAC ACC AAC CCC) that anneal to the origin of replication of both strands of the minicircle, to amplify the conserved region of $120 \mathrm{bp}$ (Dish et al. 2005).

PCR was carried out in a total volume of $10 \mu \mathrm{L}$ containing 0.75 units Taq DNA polymerase in $10 \mathrm{mM}$ Tris$\mathrm{HCl}, 50 \mathrm{mM} \mathrm{KCl}, 2 \mathrm{mM} \mathrm{MgCl}, 0.4 \mathrm{mM}$ of each nucleotide (Promega Corporation) and $0.6 \mu \mathrm{M}$ of each primer (Bioneer). Forty amplification cycles were conducted, using annealing conditions of $63^{\circ} \mathrm{C}$ for $30 \mathrm{sec}, 30 \mathrm{sec}$ at $72^{\circ} \mathrm{C}$ for primer extension and $30 \mathrm{sec}$ at $95^{\circ} \mathrm{C}$ for denaturation. Each amplification experiment included one negative DNA extraction control, one negative PCR control (no DNA) and a positive PCR control for Leishmania sp. (30 fg of DNA).

Typing by RFLP - PCR products were digested individually with 10 units of the restriction enzyme HaeIII (Promega, USA) for $3 \mathrm{~h}$ at $37^{\circ} \mathrm{C}$. The resulting restriction fragments were analyzed by polyacrylamide gel electrophoresis in 1X Tris-borate-EDTA buffer, according to Volpini et al. (2004).

Statistical analysis - The data were analyzed using Epi-Info, version 6.04. The chi-square test was used to compare the proportions by gender distribution. Additionally, the one-tailed t-test was used for the comparison of mean disease duration, number of lesions and skin-test diameter. The significance level was set at $5 \%$. 
Ethical issues - This work was in accordance with resolution 196/96 of the National Health Council of the Ministry of Health of Brazil, which regulates research in human beings and has been approved by the Research Ethical Committee of Instituto René Rachou-Fiocruz (protocol 20/2006).

\section{RESULTS}

The distribution according to sex, age, number of lesions and disease duration of the 76 patients enrolled in this study demonstrated that $54(71 \%)$ patients were men. The mean age of the patients was $35.3 \pm 17.2$ years. The average number of skin lesions was 1.1 and ranged from one-five. The average of disease duration was 4.8 \pm 2.4 months.

Positive cultures were observed in 53 patients $(69.7 \%$ of the study participants). Cultures from 45 patients (59.2\% of the participants) were identified as positive by the first week, cultures from eight patients $(10.5 \%$ of the participants) were identified as positive at 14 days and at 21 days, no new positive results were observed (Table I).

The 53 patients with positive aspirate cultures had a mean age of $32.5 \pm 15.0$ years (Table II). This is younger than the mean age of the patients with negative cultures, which was $40.8 \pm 15.4$ years $(\mathrm{p}=0.02)$. Among the positive patients, 39 ( $73.6 \%$ of the participants) were men and 14 (26.4\% of the participants) were women. In the negative group, 15 (65.2\% of the participants) were men and eight (34.8\% of the participants) were women $(\mathrm{p}=0.29)$. The mean duration of disease among patients with positive cultures $(4.4 \pm 2.3$ months) was significantly lower $(p=0.009)$ than the duration of the disease in patients

TABLE I

Positivity of the lesion culture aspirate with 7, 14 and 21 days of culture of 76 patients with cutaneous leishmaniasis

\begin{tabular}{lcc}
\hline Days of culture & $\begin{array}{c}\text { Positive } \\
\mathrm{n}\end{array}$ & $\begin{array}{c}\text { Positivity } \\
\%\end{array}$ \\
\hline 7 & 45 & 59.2 \\
14 & 8 & 10.5 \\
21 & 0 & 0 \\
\hline Total & $53 / 76$ & 69.7 \\
\hline
\end{tabular}

with negative cultures (5.8 \pm 2.5 months). No differences were observed in the number of lesions in the culturepositive and culture-negative groups $(1.1 \pm 0.3$ and 1.4 \pm 1.1 lesions, respectively; $\mathrm{p}=0.55$ ) or in diameter of the skin test induration $(3.3 \pm 2.7 \mathrm{~cm}$ and $3.4 \pm 2.4 \mathrm{~cm}$, respectively; $p=0.22$ ) (Table II).

Table III shows the positivity rate of Leishmania spp. in the aspirate cultures from patients with positive and negative skin tests and the microscopic examination of the imprint smear of biopsy fragments. Among the 76 patients, 63 had a positive skin test and 13 patients had a negative skin test. The detection rate of Leishmania spp. in the aspirates from this group of patients was $63.5 \%$ and $100 \%$, respectively. The imprint smear was positive in 48 of the 76 patients (63.2\%) and negative in 28 . The aspiration culture was positive in 36 of the $48(75 \%)$ that had a positive imprint smear and in 17 of the $28(60.7 \%)$ with negative imprint smear.

Among the 76 patients studied, samples from 60 participants $(79 \%)$ were evaluated by PCR-RFLP (Table IV).

\section{TABLE III}

Positivity of the aspirate culture by skin test and imprint smear results in patients with cutaneous leishmaniasis

\begin{tabular}{lcccc}
\hline & & & \multicolumn{2}{c}{$\begin{array}{c}\text { Culture aspirate } \\
\text { positivity }\end{array}$} \\
\cline { 4 - 5 } Diagnostic test & Status & $\mathrm{n}$ & $+/ \mathrm{n}$ & $\%$ \\
\hline Montenegro skin test & Positive & 63 & $40 / 63$ & 63.5 \\
& Negative & 13 & $13 / 13$ & 100 \\
Imprint Smear & Positive & 48 & $36 / 48$ & 75 \\
& Negative & 28 & $17 / 28$ & 60.7 \\
\hline
\end{tabular}

TABLE IV

Sample pattern obtained by PCR-RFLP assay

\begin{tabular}{lccc}
\hline & \multicolumn{2}{c}{ Aspirate culture } & \\
\cline { 2 - 3 } PCR-RFLP profile & Positive & Negative & \\
\hline L. (V.) braziliziensis & 36 & 09 & $45(100)$ \\
L. (L.) amazonensis & 01 & 03 & $04(8)$ \\
\hline
\end{tabular}

TABLE II

Age and sex distribution, disease duration, number of lesions and skin test results among patients with positive and negative aspirate cultures

\begin{tabular}{lccc}
\hline Variable & $\begin{array}{c}\text { Positive culture } \\
(\mathrm{n}=53)\end{array}$ & $\begin{array}{c}\text { Negative culture } \\
(\mathrm{n}=23)\end{array}$ & $\mathrm{p}$ value \\
\hline Mean age (years) & $32.5 \pm 15,0$ & $40.8 \pm 15,4$ & 0.02 \\
Male & $39(73.6 \%)$ & $15(65.2 \%)$ & 0.007 \\
Female & $14(26.4 \%)$ & $8(34.8 \%)$ & 0.29 \\
Mean of disease duration (months) & $4.4 \pm 2.3$ & $5.8 \pm 2.5$ & 0.009 \\
Mean of number of lesions & $1.1 \pm 0.3$ & $1.4 \pm 1.1$ & 0.55 \\
Mean the skin-test diameter $(\mathrm{cm})$ & $3.3 \pm 2.7$ & $3.4 \pm 2.4$ & 0.22 \\
\hline
\end{tabular}


From these 60 patients, the PCR was positive in $53(80 \%)$ of the samples and negative in seven. We were able to perform PCR-RFLP analysis for 49 of these 60 samples. We observed a pattern compatible with $L$. (V.) braziliensis in $45(92 \%)$ samples and in the remaining four samples the pattern observed was compatible with $L$. (L.) amazonensis.

The combined methods (smears and aspirates cultures) or (aspirates and PCR) improved the detection rate of Leishmania spp. to $85.5 \%(65 / 76)$ and $98.3 \%$ $(59 / 60)$, respectively.

\section{DISCUSSION}

The results observed in this work showed a high detection rate of Leishmania spp. of in the CL lesion aspirate cultures, confirming the results obtained by Romero et al. (1999). The benefit of this technique is its applicability in routine practices, targeting field sites. Another important advantage is the possibility that sample collection can be performed by well-trained health workers, in contrast with the biopsy procedure that ideally requires a trained physician.

The correct diagnosis and characterization of the infecting Leishmania species may be important for evaluating prognosis and prescribing the correct treatment. In the present work, promastigotes were observed in the majority of the cultures by microscopic examination within one week of sample cultivation. Similar results were obtained using a sensitive microcapillary culture method for the diagnosis of CL in Turkey (Allahverdiyev et al. 2004). These authors demonstrated that the average period of incubation needed to detect promastigotes was two-seven days, thereby allowing for the correct diagnosis and treatment of the patient within a few days.

Under the conditions of the present study, all cultures were positive within two weeks. It is possible that the optimization of the culturing methods may result in a decrease in this time. Shaw and Lainson (1981) demonstrated that the use of the same agar base with a different type of overlay, may influence the growth of the parasite. The growth of two stocks of L. braziliensis guyanensis, isolated from a cutaneous infection contracted in a region of Brazil, was good in NNN medium with an overlay of condensation fluid or Schneider's medium. Nevertheless, the growth of the parasite using NNN with an overlay of saline with condensation fuid was more sensitive than Schineider's medium and also better than NNN with an overlay of saline or Locke's solution.

Furthermore, the replacement of saline with RPMI medium in a microculture procedure for the isolation of Leishmania parasites demonstrated sensitivity as high as $80 \%$ compared with $66.7 \%$ obtained with saline (Ihalamulla et al. 2006).

Characteristics such as age, sex, disease duration and diameter of the induration in the Montenegro skin test influenced the outcome of the cultures. The detection rate of Leishmania spp. of the aspiration culture was higher in young male patients (average age 32.5 year old) with a history of disease duration of four months or less. Parasite loads tend to decrease with time, mak- ing diagnosis difficult using parasitological methods. Disease diagnosis may therefore benefit from the more sensitive molecular assays. Weigle et al. (2002) demonstrated that PCR analysis of biopsies from chronic CL lesions was twice as sensitive compared to three conventional methods (Giemsa-stained lesion scrapings, the culture of lesion aspirates and macerated biopsies in Senekjie's media).

The reported positivity rate of Leishmania spp. observed by microscopic examination of imprint smears for the presence of parasites usually ranges from $20 \%$ to $40 \%$ (Salinas et al. 1989). Nevertheless, in this work, positive smears were observed in 48/76 (63.16\%) patients. This high positivity may be attributed to the fact that three smears were prepared from each biopsied lesion. The detection rate of Leishmania spp. of the aspirate in this group of positive smears was $69.8 \%$.

The contamination rate of the cultures was very low (data not shown) and did not influence the visualization of the parasite.

The PCR-RFLP analysis was performed using samples from the stored slides of Giemsa-stained imprints from each patient. PCR is a sensitive tool for the detection of target DNA and offers an effective method to confirm suspected cases of leishmaniasis using samples that have been collected by simple methods (even archived samples) (Weigle et al. 2002, Brustoloni et al. 2007). The use of samples from archived slides is an interesting approach which may be an alternative method for detection and identification of Leishmania parasite. DNA was isolated from 92 Giemsa-stained smears of lesions from suspected cases of CL and used for PCR-based diagnosis of Leishmania infection. Although the smears had been stored for up to four years, all the microscopypositive slides were also positive by PCR and four of the 14 smears that were negative by microscopy (although of lesions that were clinically consistent with leishmaniasis) were also PCR-positive (Motazedian et al. 2002).

Volpini et al. (2006) confirmed that Leishmania DNA can be amplified by PCR from slides archived for up to 36 years. These authors highlighted the historical value of stored tissue collections for retrospective studies, which may be useful in predicting the course of many diseases (Volpini et al. 2006). Brustoloni et al. (2007) demonstrated that it is possible to reach a retrospective diagnosis of visceral leishmaniasis from stored Giemsa-stained bone marrow slides. Slides can be easily stored and do not require low temperature for conservation of the DNA.

The identification of the Leishmania species causing CL using PCR-RFLP has been performed by several investigators. Using the PCR-RFLP methodology on biopsies of patients from an endemic area in $\mathrm{MG}$, Volpini et al. (2004) demonstrated the potential value of this technique in the diagnosis and identification of Leishmania species causing CL. The PCR-RFLP method was also validated by the identification of Leishmania species in a total of 475 slides obtained from an outpatient clinic located in a region of endemic CL in MG that had been stored for up to 36 years. The Leishmania sp. DNA was amplified from $395 / 475(83.2 \%)$ of the DNA samples using primers specific for the minicircle kinetoplast 
sequence and $L$. (V.) braziliensis was the only species present in these samples (Volpini et al. 2006). Using the PCR-RFLP methodology we observed a pattern compatible with $L .(V$.) braziliensis in the majority of the samples. This parasite species is largely responsible for ATL in Southeastern Brazil.

Given the feasibility and the high positivity of the aspirate culture, we believe that this procedure could be routinely adopted for $L$. (V.) braziliensis isolation from CL lesions.

\section{REFERENCES}

Allahverdiyev AM, Uzun S, Bagirova M, Durdu M, Memisoglu HR 2004. A sensitive new microculture method for diagnosis of cutaneous leishmaniasis. Am J Trop Med Hyg 70: 294-297.

Berman JD 1997. Human leishmaniasis: clinical, diagnostic and chemotherapeutic developments in the last 10 years. Clin Infect Dis 24: 684-703.

Brustoloni YM, Lima RB, da Cunha RV, Dorval ME, Oshiro ET, de Oliveira AL, Pirmez C 2007. Sensitivity and specificity of polymerase chain reaction in Giemsa-stained slides for diagnosis of visceral leishmaniasis in children. Mem Inst Oswaldo Cruz 102: 497-500.

Chang KP, Fish WR 1983. Leishmaniasis. JB Jensen (ed.). In In vitro cultivation of pathogenic protozoa of man and domestic animals, FL: CRC Press, Boca Raton, p. 111-153.

Desjeux P 2004. Leishmaniasis. Nat Rev Microbiol 2: 692-693.

Disch J, Pedras MJ, Orsini M, Pirmez C, de Oliveira MC, Castro M, Rabello A 2005. Leishmania (Viannia) subgenus kDNA amplification for the diagnosis of mucosal leishmaniasis. Diagn Microbiol Infect Dis 51: 185-90.

Grimaldi G Jr, Tesh RB, McMahon-Pratt D 1989. A review of the geographic distribution and epidemiology of leishmaniasis in the New Word. Am J Trop Med Hyg 41: 687-725.

Ihalamulla RL, Rajapaksa US, Karunaweera ND 2006. Microculture for the isolation of Leishmania, modified to increase efficacy: a follow-up to a previous study. Ann Trop Med Parasitol 100: 87-89.

Marzochi MC, Teixeira PC, Marzochi KB, da Conceição NF, Coutinho W, de Brito DB 1993. Vacuum aspiratory puncture system for Leishmania culturing, isolation and transport. Rev Inst Med Trop Sao Paulo 35: 301-303.

Motazedian H, Karamian M, Noyes HA, Ardehali S 2002. DNA extraction and amplification of leishmania archived, Giemsa stained slides, for the diagnosis of cutaneous leishmaniasis by PCR. Ann Trop Med Parasitol 96: 31-34.

MS - Ministério da Saúde 2007. Manual de vigilância da leishmaniose tegumentar americana, $2^{\mathrm{a}}$ edição. Available from: http://portal. saude.gov.br/portal/arquivos/pdf/manual_tta_2ed.pdf.

Pirmez C, da Silva Trajano V, Paes-Oliveira Neto M, da-Cruz AM, Gonçalves-da-Costa SC, Catanho M, Degrave W, Fernandes O 1999. Use of PCR in diagnosis of human american tegumentary leishmaniasis in Rio de Janeiro, Brazil. J Clin Microbiol 37: 1819-1823.

Reed SG 1996. Diagnosis of leishmaniasis. Clin Dermatol 14: 471-478.

Reithinger R, Davies CR 1999. Is the domestic dog (Canis familiaris) a reservoir host of American cutaneous leishmaniasis? A critical review of the current evidence. Am J Trop Med Hyg 61: 530-534.

Romero GA, Guerra MV, Paes MG, Macêdo VO 2001. Comparison of cutaneous leishmaniasis due to Leishmania (Viannia) braziliensis and $L$. (V.) guyanensis in Brazil: therapeutic response to meglumine antimoniate. Am J Trop Med Hyg 65: 456-465.

Romero GA, Sampaio RN, Macedo V de O, Marsden PD 1999. Sensitivity of a vacuum aspiratory culture technique for diagnosis of localized cutaneous leishmaniasis in an endemic area of Leishmania (Viannia) braziliensis transmission. Mem Inst Oswaldo Cruz 94: 505-508.

Salinas G, Valderrama L, Palma G, Montes G, Saravia NG 1989. Detection of amastigotes in cutaneous and mucocutaneous leishmaniasis using the immunoperoxidase method, using polyclonal antibody: sensibility and specificity compared with conventional methods of diagnosis. Mem Inst Oswaldo Cruz 84: 53-60.

Shaw JJ, Lainson R 1981. The in vitro cultivation of members of the Leishmania braziliensis complex. Trans $R$ Soc Trop Med Hyg 75: 127.

Sokal JE 1975. Measurement of delayed skin-test responses. $N$ Engl J Med 293: 501-502.

Volpini AC, Marques MJ, Lopes dos Santos S, Machado-Coelho GL, Mayrink W, Romanha AJ 2006. Leishmania identification by PCR of Giemsa-stained lesion imprint slides stored for up to 36 years. Clin Microbiol Infec 12: 815-818.

Volpini AC, Passos VMA, Oliveira GC, Romanha AJ 2004. PCRRFLP to identify Leishmania (Viannia) brasiliensis and $L$. (Leishmania) amazonensis causing American cutaneous leishmaniasis. Acta Trop 90: 31-37.

Weigle KA, Labrada LA, Lozano C, Santrich C, Barker DC 2002. PCR-based diagnosis of acute and chronic cutaneous leishmaniasis caused by Leishmania (Viannia). J Clin Microbiol 40: 601-606. 